\title{
EL DEFENSOR DEL PUEBLO EUROPEO Y EL TRATADO DE LISBOA ${ }^{1}$
}

\author{
P. NIKIFOROS DIAMANDOUROS \\ Defensor del Pueblo Europeo
}

\author{
SUMARIO \\ I. Introducción. \\ II. La ciudadanía de la Unión antes del Tra- \\ tado de Lisboa. \\ III. El Tratado de Lisboa. \\ IV. El Defensor del Pueblo Europeo. \\ V. Conclusión.
}

\section{INTRODUCCIÓN}

En 2009 se cumplió el bicentenario de la institución del Defensor del Pueblo y, para celebrar este acontecimiento, en el mes de junio nos reunimos en Estocolmo defensores del pueblo de todo el mundo². Desde la perspectiva

1 El presente artículo se basa en la conferencia anual Jean Monnet que el Defensor del Pueblo Europeo impartió en la Universidad de Hull el 18 de marzo de 2010. La conferencia se ha revisado y publicado en inglés en el sitio web de la Universidad de Hull en forma de documento de trabajo del Centro de Estudios de la Unión Europea.

2 La figura del Ombudsman o Defensor del Pueblo tiene su origen en Suecia, cuyo Parlamento nombró el primer Defensor del Pueblo parlamentario del mundo en 1809. Las funciones de la institución eran supervisar la actividad de los órganos jurisdiccionales y de otros poderes públicos, tramitar las reclamaciones de los ciudadanos y denunciar a los funcionarios y ministros que actuaran de forma ilícita. En los ciento cincuenta años siguientes, sólo dos países más crearon una institución del Defensor del Pueblo con competencia general, a saber: Finlandia en 1919 y Dinamarca en 1955. El modelo danés difería de los antecesores sueco y finlandés en que el Defensor del Pueblo no estaba facultado para supervisar la labor de los órganos jurisdiccionales ni para actuar como fiscal. En la versión danesa, las funciones de la institución son investigar e informar sobre las reclamaciones presentadas contra los poderes públicos, con exclusión de los órganos jurisdiccionales, pero sin competencia para tomar decisiones jurídicamente vinculantes.

Durante la segunda mitad del siglo XX, la institución del Defensor del Pueblo se extendió por todo el mundo y hoy en día continúan surgiendo nuevas oficinas. Según el Instituto Internacional del Defensor del Pueblo, la asociación profesional que representa a los defensores del pueblo a 
del Defensor del Pueblo Europeo, 2009 fue testigo del comienzo de nuestro decimoquinto año de funcionamiento. También marcó el final de mi primer mandato completo como Defensor del Pueblo. La decisión adoptada por el Parlamento Europeo el 20 de enero de este año de reelegirme para un segundo mandato constituye, a mi parecer, la aprobación del trabajo que nuestra oficina ha realizado y nos anima a continuar luchando por una administración de la UE más abierta y responsable, con una cultura de servicio y orientada al ciudadano.

Es un momento interesante para estar al frente de esta institución. Una de las principales prioridades del Defensor del Pueblo Europeo durante los próximos cinco años será contribuir a garantizar que la Unión Europea cumpla con los beneficios prometidos a los ciudadanos por el Tratado de Lisboa. Antes de analizar en detalle las disposiciones del Tratado que resultan de mayor relevancia para los ciudadanos europeos, es importante definir el concepto concreto de "ciudadanía", recurrente en el presente artículo, y entender su evolución en la Unión a lo largo del tiempo.

\section{CiUdADANÍA}

El presente artículo se centrará en la ciudadanía entendida como conjunto de derechos y obligaciones, incluidos los derechos que podrían conducir de forma dinámica a la construcción de la identidad política. En concreto, se basa en el concepto de ciudadanía entendida como una relación en constante evolución entre las personas físicas y los encargados de ejercer el poder político. Mi visión de la función del Defensor del Pueblo Europeo incluye, como elemento esencial, la responsabilidad especial de alimentar y fomentar dicha relación. El Tratado de Maastricht creó la oficina del Defensor del Pueblo Europeo como medida para reducir la distancia existente entre los ciudadanos y las instituciones de la Unión Europea, que en aquel momento empezaba a reconocerse como un problema.

escala internacional, en la actualidad existen defensores del pueblo en unos ciento veinte países de todo el mundo.

El ritmo de evolución de la institución del Defensor del Pueblo ha resultado especialmente sorprendente en Europa. Cuando el Tratado de Maastricht creó la oficina del Defensor del Pueblo Europeo a principios de los noventa, sólo existían defensores del pueblo nacionales en una ajustada mayoría de los Estados miembros de la Unión Europea: siete de doce. Tres de los cinco Estados miembros que no disponían de esta institución, a saber, Bélgica, Grecia y Luxemburgo, crearon posteriormente una oficina del Defensor del Pueblo, y todos los países que se adhirieron a la Unión en 1995, 2004 y 2007 contaban con defensores del pueblo nacionales en el momento de su adhesión. El resultado es que, actualmente, veinticinco de los veintisiete Estados miembros tienen un Defensor del Pueblo nacional. En los dos Estados miembros que no disponen de esta institución, a saber, Alemania e Italia, existen defensores del pueblo regionales y locales. Además, la Comisión de Peticiones del Bundestag (Parlamento federal) alemán desempeña una función similar a la de un Defensor del Pueblo nacional y, de hecho, es miembro del Instituto Internacional del Defensor del Pueblo. 
Diez años más tarde, frustrados por la experiencia de la conferencia intergubernamental que elaboró el Tratado de Niza, los dirigentes europeos formularon la denominada "Declaración de Laeken". Uno de sus objetivos declarados era aumentar la legitimidad democrática y la transparencia de las instituciones de la Unión Europea.

La reciente entrada en vigor del Tratado de Lisboa marca la última fase del proceso en curso de reconocimiento y capacitación de los ciudadanos europeos. En este artículo comentaré las numerosas disposiciones del Tratado que brindan valiosas oportunidades para reducir la distancia entre los ciudadanos y los encargados de ejercer el poder político en la Unión, así como para afrontar — lo que personalmente me gusta denominar - el "déficit de legitimidad" de la Unión. Este último aspecto es importante porque la legitimidad no constituye una opción adicional, sino un requisito fundamental para la eficacia a largo plazo del proceso de integración europea.

\section{LA DIMENSIÓN DE LA “IDENTIDAD POLÍTICA" EN LA CIUDADANÍA}

Conviene completar lo que he señalado anteriormente sobre la idea de ciudadanía basada en derechos y obligaciones y su relación con la identidad política. Los derechos y obligaciones constituyen, sin duda, conceptos jurídicos, pero un mero análisis jurídico de los mismos daría una visión empobrecida de la ciudadanía. De hecho, una de las concepciones más influyentes de la ciudadanía basada en derechos y obligaciones es la del sociólogo T. H. Marshall, que modeló la evolución de la ciudadanía británica como un proceso histórico acumulativo, que comenzaba con los derechos civiles, seguía con los políticos y culminaba con los derechos sociales ${ }^{3}$.

Por ahora, nos centraremos en los derechos políticos y, en concreto, en su finalidad: ¿para qué sirven estos derechos?

En mi opinión, la pregunta tiene una doble respuesta. En primer lugar, los derechos políticos permiten a los ciudadanos exigir responsabilidades a los poderes públicos por sus acciones. En segundo lugar, les permiten también participar en actividades públicas y en la vida política, con miras a influir en la forma de gobierno. Desde este punto de vista, la evolución de la ciudadanía consiste en aumentar la calidad de la democracia y reforzar la legitimidad de las instituciones políticas democráticas, tal como dispuso la Declaración de Laeken para la Unión hace casi una década.

Dicha Declaración hizo también hincapié en el principio de transparencia, que resulta fundamental por lo que respecta a la responsabilidad y la participación. La idea básica de la transparencia es que los ciudadanos deberían poder obtener fácilmente información sobre las actividades pasadas, presentes y futuras de los poderes públicos. Los ciudadanos necesitan conocer esta in-

3 MARSHALL, T. H.: Citizenship and Social Class and Other Essays, Cambridge University Press, Cambridge, 1950. 
formación para poder exigir responsabilidades a los poderes públicos y poder participar de forma efectiva en el debate público actual que forma parte de una democracia sana.

Por tanto, transparencia, responsabilidad y participación van de la mano y son aspectos indispensables para fomentar la confianza de los ciudadanos en los poderes públicos oportunos.

\section{LA CIUDADANÍA DE LA UNIÓN ANTES DEL TRATADO DE LISBOA}

\section{ANTES DE MAASTRICHT}

A continuación, describiremos brevemente la trayectoria de los ciudadanos europeos en la Unión desde los inicios de las Comunidades Europeas. En aquel momento, se hacía más hincapié en las cuestiones económicas que en los derechos y obligaciones individuales. No se abordaban en absoluto de forma explícita las nociones de transparencia y legitimidad.

No obstante, a principios de los sesenta comenzó a esclarecerse que las Comunidades eran algo más que una simple organización internacional. De hecho, los Tratados habían creado poderes legislativos y ejecutivos supranacionales con facultades para otorgar derechos e imponer obligaciones a personas físicas ${ }^{4}$. Además, la confirmación de que el Derecho de la UE disfruta de primacía o supremacía sobre el Derecho nacional ${ }^{5}$ vino acompañada de esfuerzos — sobre todo de la mano del Tribunal de Justicia - para salvaguardar los derechos individuales derivados del Derecho de la Unión.

Tras haber establecido que el Derecho de la Unión confiere derechos a las personas físicas, el Tribunal resolvió que tales derechos incluyen otros fundamentales, que tanto las instituciones de la Unión como las autoridades nacionales deben respetar cuando apliquen dicho Derecho ${ }^{6}$.

Por supuesto, el Tribunal de Justicia no era el único que trataba de proteger y promover los derechos de los ciudadanos. Cada modificación de los Tratados ha traído consigo el enriquecimiento de los derechos específicos de los ciudadanos de la Unión y se ha centrado en los principios esenciales para la capacitación de los ciudadanos, a saber, la transparencia y la responsabilidad.

4 Véase el asunto 26/62, NV Algemene Transporten Expeditie Onderneming contra Nederlandse Administratie der Belastingen [1963] Rec. 1.

5 Véase el asunto 6/64, Costa contra ENEL [1964] Rec. 585.

6 Véase el asunto 11/70, International Handelsgesellschaft mbH contra Einfuhr etc. [1970] Rec. 1125, el asunto 4/73, Nold KG contra la Comisión [1974] Rec. 491, y el asunto 5/88, Wachauf [1989] Rec. 2609. 


\section{El Tratado de MaAstricht}

En este contexto, merece especial atención el Tratado de Maastricht. Este Tratado introdujo la ciudadanía de la Unión ${ }^{7}$. Entre los derechos especiales de los ciudadanos europeos se encuentran el derecho a la libertad de circulación y residencia, el derecho de sufragio en las elecciones al Parlamento Europeo y en las elecciones locales, el derecho de reclamar protección diplomática y consular, el derecho de formular peticiones y el derecho de recurrir al Defensor del Pueblo Europeo.

El Tratado de Maastricht ponía también de relieve el principio de subsidiariedad con vistas a garantizar que las decisiones se tomaran de la forma más próxima posible a los ciudadanos. También fue el primer Tratado que mencionó de forma específica el principio de transparencia ${ }^{8}$. Estas medidas se introdujeron, en parte, en un intento de contrarrestar el denominado "déficit democrático" o "déficit de legitimidad", tal como lo he llamado anteriormente.

\section{Después De MAAstricht}

El Tratado de Ámsterdam de 1997 abordó el problema de legitimidad de la Unión mediante una mayor transparencia. El Tratado disponía que las decisiones de la Unión debían tomarse de la forma más abierta posible y establecía el derecho de acceso de los ciudadanos y residentes de la Unión a los documentos?

El Tratado de Niza, acordado a finales del año 2000, se centraba en cuestiones institucionales. Contenía pocos o ningún aspecto que afectara a los ciudadanos, y la conferencia intergubernamental que lo había negociado reconoció que seguía sin resolverse el problema de legitimidad. Un año más tarde, la Declaración de Laeken afirmaba que la gente deseaba unas instituciones europeas:

"menos lentas y rígidas y, sobre todo, más eficientes y transparentes. Muchos piensan también que la Unión debería prestar mayor atención a sus preocupaciones concretas en lugar de intervenir en los más mínimos detalles en asuntos que, por su propia naturaleza, sería mejor poner en manos de los representantes electos de los Estados miembros y de las regiones. Algunos sienten incluso esta situación como una amenaza a su identidad. Pero, lo que es quizás aún más importante: los ciudadanos consideran que las cosas se hacen demasiado a menudo a sus espaldas y desean un mayor control democrático" ${ }^{10}$.

7 Actualmente, el artículo 20 del TFUE.

8 Declaración $n .^{\circ} 17$ adjunta al Tratado.

9 El derecho de acceso del público se encuentra actualmente consagrado en el artículo 15 del TFUE.

10 Conclusiones de la Presidencia del Consejo Europeo de Laeken (14 y 15 de diciembre de 2001): Anexo I: Declaración de Laeken sobre el futuro de la Unión Europea, en Boletín de la Unión Europea, 2001, n. ${ }^{\circ}$ 12, págs. 19-23. 
Aunque éste pudiera parecer un diagnóstico bastante claro del problema, considero justo afirmar que, desde Laeken, no hemos avanzado lo suficiente con respecto al cumplimiento de estas exigencias.

- La Convención Europea que elaboró el Proyecto de Tratado por el que se instituye una Constitución para Europa fue transparente, pero sus participantes se centraron más en los problemas internos que en los ciudadanos.

- Los referendos de Francia y los Países Bajos impidieron su ratificación.

- El Tratado de Lisboa retomó el método intergubernamental, cuyas deficiencias condujeron a la Declaración de Laeken.

- Los dos referendos irlandeses generaron un auténtico debate entre los ciudadanos del país. Pero fuera de Irlanda, es probable que el proceso de ratificación de Lisboa haya tenido, en general, un impacto negativo sobre la percepción de la legitimidad de la Unión Europea y sus instituciones.

No obstante, aunque el proceso de ratificación no glorificara ni mucho menos a la Unión, la entrada en vigor del Tratado de Lisboa representa una auténtica oportunidad para lograr verdaderos avances.

\section{EL TRATADO DE LISBOA}

\section{Una Carta de los Derechos Fundamentales jurídicamente vinculante}

Una de las mejoras más significativas para los ciudadanos que ha traído consigo el Tratado de Lisboa es que la Carta de los Derechos Fundamentales tiene ahora el mismo valor jurídico que los Tratados ${ }^{11}$.

Cabe mencionar que la Carta ha existido desde el año 2000, cuando fue proclamada por primera vez en la cumbre, celebrada en Niza, en que se acordó el Tratado omónimo. Su redacción corrió a cargo de una Convención integrada principalmente por representantes de Jefes de Estado o de Gobierno, del Parlamento Europeo y de los Parlamentos nacionales. El Defensor del Pueblo Europeo participó en la Convención en calidad de observador oficial y, como explicaré más adelante, propuso con éxito que la Carta incluyera el derecho fundamental a una buena administración.

La Carta reúne derechos ya contenidos en la jurisprudencia del Tribunal de Justicia de la Unión Europea y en una serie de textos, incluidos el Convenio Europeo de Derechos Humanos, la Carta Social Europea del Consejo de Europa, la Carta Comunitaria de los Derechos Sociales Fundamentales de los Trabajadores y las citadas disposiciones del antiguo Tratado CE sobre los

11 En un principio, la Carta se proclamó en diciembre de 2000 y se firmó y proclamó de nuevo el 12 de diciembre de 2007 antes de la firma del Tratado de Lisboa el 13 de diciembre de 2007, Diario Oficial C 303 de 14 de diciembre de 2007, pág. 1. 
derechos de los ciudadanos. Se trata de un documento más amplio que el Convenio Europeo de Derechos Humanos, ya que, además de los clásicos derechos civiles y políticos, incluye derechos económicos y sociales, como el derecho a la atención sanitaria, los derechos de las personas mayores y la protección de la familia. Varias de sus disposiciones abordan de forma específica algunas cuestiones modernas, como la bioética y la protección de los datos de carácter personal.

Las disposiciones de la Carta van dirigidas a las instituciones de la Unión y a los Estados miembros cuando apliquen el Derecho de la Unión.

\section{EL DERECHO A UNA BUENA ADMINISTRACIÓN}

El Defensor del Pueblo Europeo ha aplicado la Carta en su trabajo desde su proclamación en Niza en diciembre de 2000. Entre las disposiciones que han demostrado ser de especial interés por lo que se refiere a la supervisión de las instituciones de la UE son las relativas a la igualdad (Título III de la Carta) y, por supuesto, las relativas a la ciudadanía (Título V).

En la categoría de ciudadanía, la Carta incluye el derecho a recurrir al Defensor del Pueblo (artículo 43), el derecho a formular peticiones al Parlamento Europeo (artículo 44) y el derecho a una buena administración (artículo 41). Permítanme centrarme en este último derecho fundamental.

El derecho a una buena administración se incluyó en la Carta de los Derechos Fundamentales a raíz de una propuesta presentada por el primer Defensor del Pueblo Europeo, Jacob Söderman. Para ayudar a respaldar su propuesta, el Defensor del Pueblo se remitió al hecho de que la Convención tenía la posibilidad de tener en cuenta los avances producidos en las normas de derechos humanos y en la relación entre los ciudadanos y la administración pública. Afirmó que entre estos avances se encontraba, ante todo, la idea de que los ciudadanos tienen derecho a una tramitación correcta, justa y diligente de sus asuntos por una administración pública abierta, responsable y dotada de sentido de servicio público.

La Carta estipula el derecho fundamental a una buena administración de la siguiente manera: 1 . Toda persona tiene derecho a que las instituciones y órganos de la Unión traten sus asuntos imparcial y equitativamente y dentro de un plazo razonable. Este derecho incluye en particular: a) el derecho de toda persona a ser oída antes de que se tome en contra suya una medida individual que le afecte desfavorablemente; b) el derecho de toda persona a acceder al expediente que le afecte, dentro del respeto de los intereses legítimos de la confidencialidad y del secreto profesional y comercial; c) la obligación que incumbe a la administración de motivar sus decisiones. 3. Toda persona tiene derecho a la reparación por la Comunidad de los daños causados por sus instituciones o sus agentes en el ejercicio de sus funciones, de conformidad con los principios generales comunes a los Derechos de los Estados miembros. 4. Toda persona podrá dirigirse a las instituciones de la Unión en 
una de las lenguas de los Tratados y deberá recibir una contestación en esa misma lengua.

Se prevé que la naturaleza jurídicamente vinculante de la Carta y la consecuente posibilidad de protección judicial de las personas físicas aumenten las repercusiones del derecho a una buena administración. Asimismo, se prevé una intensificación de los esfuerzos del Defensor del Pueblo para fomentar una buena administración en interés público y buscar soluciones extrajudiciales a los problemas de los ciudadanos.

Si pensamos en la ciudadanía como conjunto de derechos y obligaciones y como relación en constante evolución con los encargados de ejercer el poder político, el hecho de que el derecho fundamental a una buena administración se incluya en esta Carta jurídicamente vinculante constituye, sin duda, una importante fuente de estímulo.

Como ya he esbozado al principio de este artículo, otros elementos fundamentales para la citada relación entre los ciudadanos y los encargados de ejercer el poder político son la responsabilidad, la participación y, como fundamento de ambas, la transparencia. El Tratado de Lisboa presagia asimismo importantes mejoras en estos ámbitos.

\section{MAYOR APERTURA Y TRANSPARENCIA}

Varios artículos del Tratado de Lisboa estipulan una mayor apertura y transparencia en las actividades de las instituciones de la Unión:

- El apartado 3 del artículo 10 del Tratado de la Unión Europea menciona que las decisiones de la Unión "serán tomadas de la forma más abierta y próxima posible a los ciudadanos". El apartado 8 del artículo 16 es nuevo, aunque ya existía en el Tratado una disposición similar. Dicho apartado estipula que el Consejo se reunirá en público cuando delibere y cote sobre un proyecto de acto legislativo. Como Defensor del Pueblo, he alegado desde hace tiempo que la plena transparencia del proceso legislativo del Consejo debería reforzar tanto la ciudadanía nacional como la ciudadanía de la Unión. Debería permitir a los europeos conocer las actividades que realizan a escala europea los gobiernos que han elegido como ciudadanos europeos. También les permitiría, en su calidad de ciudadanos de la Unión, llevar a cabo un seguimiento más eficaz de la labor de una institución esencial de aquélla y promover así la responsabilidad.

- El artículo 42 de la Carta de los Derechos Fundamentales y el artículo 15, apartado 3, del Tratado de Funcionamiento de la Unión Europea amplían el derecho de acceso del público a los documentos, que ahora es aplicable a todas las instituciones, órganos y organismos de la Unión, incluido el Consejo Europeo. Dado el elevado número de investigaciones sobre falta de transparencia (36\% de las investi- 
gaciones de 2009) que lleva a cabo el Defensor del Pueblo cada año, haré especial hincapié en este derecho fundamental.

- El apartado 1 del artículo 15 del Tratado de Funcionamiento de la Unión Europea también es nuevo. Establece que "a fin de fomentar una buena gobernanza y de garantizar la participación de la sociedad civil, las instituciones [...] de la Unión actuarán con el mayor respeto posible al principio de apertura". Desde mi punto de vista, esta nueva disposición tiene suma importancia porque exige a las instituciones que tomen la iniciativa por lo que respecta a garantizar la apertura de su labor.

- Permítanme poner un ejemplo de lo que esto podría suponer en la práctica. A finales del año pasado, la administración federal estadounidense aprobó una "Directiva de Gobierno abierto", que, entre otras cosas, pide a todos los organismos que i) publiquen información en Internet que incluya, al menos, tres bases de datos de formato abierto de alto valor, y que ii) elaboren y publiquen un Plan de Gobierno Abierto para mejorar la transparencia e integrar la participación y la colaboración del público en sus actividades. Es cierto que existen grandes diferencias entre los organismos federales de los Estados Unidos y los organismos de la Unión. Sin embargo, cabría reflexionar en qué medida podrían aplicarse los principios de esta Directiva estadounidense en el contexto de la Unión Europea.

\section{PARTICIPACIÓN}

La introducción de un nuevo modo de participación del público en la vida democrática de la Unión en forma de "iniciativa ciudadana" debería contribuir en gran medida a la capacitación de los ciudadanos europeos. La Iniciativa Ciudadana Europea ${ }^{12}$ estipula que un grupo de un millón de ciudadanos que sean nacionales de un número significativo de Estados miembros podrá pedir directamente a la Comisión Europea que presente propuestas legislativas en un ámbito de competencia de la Unión Europea.

En enero de 2010, respondí a la consulta pública de la Comisión Europea sobre la forma en que debía funcionar en la práctica la iniciativa ciudadana ${ }^{13}$. Traté de anticipar las dudas que podrían surgir durante su aplicación, sobre todo aquéllas que podrían dar lugar a la presentación de reclamaciones ante el Defensor del Pueblo. Con la identificación anticipada de estas dudas y la propuesta de respuestas efectivas pretendía fomentar la buena administración de la Comisión Europea y contribuir así al éxito de este nuevo y valioso instrumento.

12 Artículo 11 del Tratado de la Unión Europea.

13 La contribución del Defensor del Pueblo a la consulta pública puede consultarse en la siguiente dirección: http://www.ombudsman.europa.eu/resources/otherdocument.faces/es/4592/ html.bookmark 
En mi opinión, el derecho de un millón de ciudadanos a formular peticiones a la Comisión debería implantarse de forma que capacite realmente a los ciudadanos. En otras palabras, el acto legislativo que lo ponga en vigor no debería redactarse de forma que convierta estas peticiones en un recurso más para la Comisión. En concreto, deberían participar también los Parlamentos nacionales y el Parlamento Europeo.

El Tratado de Lisboa exige asimismo a las instituciones de la UE que i) den a los ciudadanos y a las asociaciones representativas la posibilidad de expresar e intercambiar públicamente sus opiniones en todos los ámbitos de actuación de la Unión, y que ii) mantengan un diálogo abierto, transparente y regular con las asociaciones representativas y la sociedad civil.

Estas disposiciones resultan muy significativas porque el Derecho de la Unión afecta a cada uno de los ciudadanos de cada Estado miembro. La forma en que los ciudadanos perciben el Derecho de la Unión como fuente de capacitación o restricción, de beneficios o cargas y si consideran que tienen voz por lo que respecta a su aprobación constituyen aspectos de suma importancia para la legitimidad de la Unión.

\section{RESPONSABILIDAD}

Con vistas al refuerzo de la legitimidad de las instituciones políticas democráticas, resulta también esencial la capacidad de los ciudadanos para exigir responsabilidades a dichas instituciones. Por lo que respecta a la Unión, el Tratado de Lisboa incrementa ciertamente la capacidad de los ciudadanos en este sentido.

Como acabo de describir, el Tratado fomenta la participación de los ciudadanos en la vida democrática de la Unión. Permítanme centrarme a continuación en el control democrático de la labor legislativa de la Unión y en lo que considero que constituye su complemento indispensable, a saber: una mayor colaboración de los ciudadanos para lograr una legislación eficaz. Después de todo, se trata del aspecto que la Declaración de Laeken identificó como ausente y marca una etapa fundamental en el camino hacia la capacitación plena de los ciudadanos.

Considero que existen otras medidas concretas que podrían adoptarse y que brindarían una buena oportunidad para ayudar a reducir la creciente desvinculación de los ciudadanos con respecto al proyecto europeo. El Tratado de Lisboa presenta un excelente punto de partida, ya que refuerza las competencias legislativas del Parlamento Europeo y de los Parlamentos nacionales. La mayor función de los Parlamentos nacionales otorga a los ciudadanos y a la sociedad civil organizada en el ámbito de los Estados miembros la posibilidad de participar en el proceso legislativo de la Unión en el marco democrático de su país, donde algunos podrán sentirse más capacitados para participar que en el ámbito de la Unión. 
Sin embargo, el proceso de elaborar actos legislativos a escala europea e incorporarlos, cuando proceda, a las legislaciones nacionales constituye tan sólo una parte de la cuestión. La legislación es —o debería ser- más que un proceso simbólico. La otra parte de la cuestión se refiere a la forma de aplicar y cumplir en la práctica la legislación. Una buena administración supone que los actos legislativos europeos deberían ser eficaces. Europa no logrará acercarse a sus ciudadanos mediante la mera promulgación de buenos actos legislativos. A menos que dichos actos se apliquen y cumplan de forma efectiva, no se obtendrán resultados provechosos.

Las autoridades de los Estados miembros tienen la responsabilidad jurídica fundamental de cumplir el Derecho de la Unión y salvaguardar los derechos que éste confiere a los ciudadanos, empresas y asociaciones. Los órganos jurisdiccionales nacionales hacen efectiva esta responsabilidad jurídica en el ámbito de cada Estado miembro al ofrecer vías de recurso - incluso contra otros poderes públicos del Estado miembro en cuestión- a aquéllos cuyos derechos han sido violados. La Comisión, en su calidad de "guardiana de los Tratados ${ }^{14}$, actúa como vigilante de la responsabilidad jurídica en el ámbito de la Unión mediante la remisión de asuntos al Tribunal de Justicia.

En cuanto a la responsabilidad política por lo que se refiere al cumplimiento y la aplicación del Derecho de la Unión, los ciudadanos tienen acceso a un mecanismo en el ámbito de la Unión mediante el derecho de petición ante el Parlamento Europeo. No obstante, lo que no existe en la mayoría de los Estados miembros es un mecanismo político para exigir responsabilidades a los poderes públicos a escala nacional en relación con los problemas que puedan surgir en el cumplimiento y la aplicación del Derecho de la Unión.

La ausencia de este tipo de mecanismo produce diversos efectos no deseados. En concreto, priva a los ciudadanos y a sus representantes de la posibilidad de examinar de forma específica y exhaustiva un importante aspecto de las actividades relacionadas con la Unión que desempeñan los poderes públicos de sus Estados miembros. Asimismo, priva al ámbito de la Unión de posible información valiosa que debería aportarse a los procesos legislativos y de elaboración de políticas con el fin de mejorar el Derecho de la Unión en el futuro.

Aunque la falta de mecanismos políticos para exigir responsabilidades a los poderes públicos en el ámbito de los Estados miembros tenga consecuencias para la Unión, ésta no está facultada para exigir su creación. Tampoco sería deseable que la Unión adquiriera esta competencia. No obstante, las instituciones de la Unión podrían y deberían fomentar y facilitar la adopción voluntaria de este tipo de mecanismos por parte de los Estados miembros.

El Parlamento Europeo podría tomar la iniciativa en este sentido, por ejemplo, seleccionando cada año uno o varios actos legislativos vigentes de la Unión para su evaluación. Entre los actos que podrían evaluarse se encuen-

14 Artículo 258 del TFUE. 
tran la Directiva sobre los derechos de los ciudadanos ${ }^{15}$, las Directivas sobre igualdad ${ }^{16} \mathrm{o}$ el Reglamento sobre los derechos de los pasajeros aéreos ${ }^{17}$.

Considero que esta propuesta de planteamiento centrado en lograr la eficacia del Derecho de la Unión con respecto a los ciudadanos ayudaría a:

- mejorar el contenido del Derecho de la Unión (y, por consiguiente, contribuiría también al objetivo de "legislar mejor");

- capacitar a los ciudadanos y a sus representantes en el Parlamento Europeo y los Parlamentos nacionales;

- cumplir las promesas y los objetivos del Tratado de Lisboa relativos a los ciudadanos;

- contribuir visiblemente a combatir el "déficit de legitimidad" de la Unión.

\section{EL DEFENSOR DEL PUEBLO EUROPEO}

La última parte del presente artículo se centrará en cómo puede el Defensor del Pueblo Europeo contribuir a reforzar la legitimidad de todo el sistema de gobernanza de la Unión Europea y, de este modo, combatir el "déficit de legitimidad".

En primer lugar, permítanme ofrecer una visión general del mandato del Defensor del Pueblo y de la forma en que éste se ha visto afectado por la entrada en vigor del Tratado de Lisboa.

\section{El mandato del Defensor del Pueblo y las modificaciones INTRODUCIDAS POR EL TRATADO DE LISBOA}

El artículo 24 del Tratado de Funcionamiento de la Unión Europea (TFUE) —antiguo artículo 21 del Tratado CE- establece el derecho a pre-

15 Directiva 2004/38/CE del Parlamento Europeo y del Consejo, de 29 de abril de 2004 relativa al derecho de los ciudadanos de la Unión y de los miembros de sus familias a circular y residir libremente en el territorio de los Estados miembros por la que se modifica el Reglamento (CEE) No 1612/68 y se derogan las Directivas 64/221/CEE, 68/360/CEE, 72/194/CEE, 73/148/CEE, 75/34/CEE, 75/35/CEE, 90/364/CEE, 90/365/CEE y 93/96/CEE, Diario Oficial L 158 de 30.4.2004, pág. 77.

16 Directiva 2000/43/CE del Consejo, de 29 de junio de 2000, relativa a la aplicación del principio de igualdad de trato de las personas independientemente de su origen racial o étnico, Diario Oficial L 180 de 19.7.2000, pág. 22, y Directiva 2000/78/CE del Consejo, de 27 de noviembre de 2000 , relativa al establecimiento de un marco general para la igualdad de trato en el empleo y la ocupación, Diario Oficial L 303 de 2.12.2000, pág. 16.

17 Reglamento (CE) n. ${ }^{\circ}$ 261/2004 del Parlamento Europeo y del Consejo, de 11 de febrero de 2004, por el que se establecen normas comunes sobre compensación y asistencia a los pasajeros aéreos en caso de denegación de embarque y de cancelación o gran retraso de los vuelos, y se deroga el Reglamento (CEE) n. ${ }^{\circ}$ 295/91, Diario Oficial L 46 de 17.2.2004, pág. 1. 
sentar reclamaciones ante el Defensor del Pueblo Europeo como uno de los derechos de ciudadanía de la Unión Europea. Como ya he mencionado anteriormente, este derecho se incluye asimismo en la Carta de los Derechos Fundamentales de la Unión Europea (artículo 43). El Defensor del Pueblo investiga posibles casos de mala administración sobre todo a partir de reclamaciones, aunque también realiza investigaciones de propia iniciativa.

La labor del Defensor del Pueblo se rige por el artículo 228 del TFUE, asî como por el Estatuto del Defensor del Pueblo ${ }^{18}$ y las disposiciones de aplicación aprobadas por el Defensor del Pueblo de conformidad con el artículo 14 del Estatuto.

Modificaciones introducidas sobre la base jurídica que rige la labor del Defensor del Pueblo como consecuencia del Tratado de Lisboa A partir de la entrada en vigor del Tratado de Lisboa, el artículo 195 del Tratado CE sobre el Defensor del Pueblo pasó a ser el artículo 228 del TFUE. El mandato del Defensor del Pueblo se ha ampliado de "las instituciones u órganos comunitarios" a "las instituciones, órganos u organismos de la Unión" ${ }^{19}$. Esta modificación tiene dos implicaciones principales: puesto que el Tratado de Lisboa suprime la estructura de pilares de la UE, el antiguo segundo pilar (Política Exterior y de Seguridad Común) se inscribe ahora en el ámbito de competencias del Defensor del Pueblo; con arreglo al artículo 13 del Tratado de la Unión Europea (TUE), el Consejo Europeo es una institución, por lo que ahora se encuentra sujeto al mandato del Defensor del Pueblo. Asimismo, cabe mencionar dos modificaciones más: el apartado 1 del artículo 228 del TFUE especifica que el Parlamento Europeo "elige" en lugar de "nombrar" al Defensor del Pueblo, mientras que el apartado 4 del mismo artículo dispone que el Estatuto del Defensor del Pueblo se establecerá mediante un Reglamento del Parlamento Europeo en vez de a través de una Decisión.

\section{REFUERZO DE LAS RELACIONES ENTRE LOS CIUDADANOS Y EL ÁMBITO DE GOBERNANZA DE LA UNIÓN}

El Tratado de Maastricht creó la institución del Defensor del Pueblo Europeo en 1993 con el fin de reforzar las relaciones existentes entre los ciudadanos y el ámbito de gobernanza de la Unión. A la vista de que las instituciones europeas se percibían como aisladas e "impersonales", tecnocráticas y

18 Decisión del Parlamento Europeo, de 9 de marzo de 1994, sobre el estatuto del Defensor del Pueblo y sobre las condiciones generales del ejercicio de sus funciones (94/262/CECA, CE, Euratom), DO L 113 de 4.5.1994, pág. 15, modificada por la Decisión del Parlamento Europeo de 18 de junio de 2008 (2008/587/CE, Euratom), Diario Oficial L 189 de 17.7.2008, pág. 25.

19 Con exclusión del Tribunal de Justicia de la Unión Europea en el ejercicio de sus funciones jurisdiccionales. El Tratado de Lisboa cambió los nombres del Tribunal de Justicia de las Comunidades Europeas y del Tribunal de Primera Instancia. Ahora se denominan conjuntamente "Tribunal de Justicia de la Unión Europea" y por separado "Tribunal de Justicia” y "Tribunal General", respectivamente. 
muy distantes de los ciudadanos de a pie, se consideró importante humanizar la administración de la Unión.

Actualmente, el Defensor del Pueblo se encuentra en su decimoquinto año de actividad. Durante este tiempo, se han llevado a cabo numerosas acciones para garantizar una administración de la Unión más abierta y responsable, con una cultura de servicio y orientada al ciudadano. El año pasado, el Defensor del Pueblo dedicó un tiempo a reflexionar sobre la misión de la institución y finalmente aprobó la siguiente formulación:

«El Defensor del Pueblo trata de lograr una solución aceptable de las reclamaciones presentadas contra las instituciones de la Unión Europea, fomenta la transparencia y promueve una cultura administrativa de servicio. Su objetivo es generar confianza a través del diálogo entre los ciudadanos y la Unión Europea y promover los más elevados niveles de comportamiento en las instituciones de la Unión".

Uno de los aspectos más importantes de la misión del Defensor del Pueblo es generar confianza a través del diálogo entre los ciudadanos y la Unión Europea. Para ello, tengo que prestar mi ayuda tanto a los ciudadanos como a las instituciones. En cuanto a estas últimas, mi objetivo es convencerlas de que, con el fin de ganarse la confianza y la aceptación del público, necesitan demostrar que son responsables y receptivas. Esto implica, entre otras cosas, estar dispuestas a explicar y justificar su actuación mediante un diálogo auténtico y positivo con los ciudadanos, tanto sobre asuntos que les afecten personalmente como sobre la forma en que se identifica y persigue el interés general.

En cuanto a los ciudadanos, mi objetivo es promover una ciudadanía activa ayudándoles tanto a ellos como a las organizaciones de la sociedad civil a conocer sus derechos y obligaciones, animándoles a participar en la vida democrática de la Unión, fomentando su capacidad de exigir responsabilidades a las instituciones europeas y reforzando su colaboración para lograr la eficacia de la legislación.

Estas medidas deberían contribuir al desarrollo de la ciudadanía europea gracias al aumento de la calidad de la democracia y al refuerzo de la legitimidad de las instituciones políticas democráticas. Por tanto, tal como espero que se desprenda de lo anterior, el Tratado de Lisboa debería facilitar en gran medida estas acciones.

\section{CONTRIBUCIÓN A GARANTIZAR QUE LA UNIÓN EUROPEA CUMPLA CON LOS BENEFICIOS PROMETIDOS A LOS CIUDADANOS POR EL TRATADO DE LisbOA}

Una de mis principales prioridades durante los próximos cinco años será contribuir a garantizar que la Unión Europea cumpla con los beneficios prometidos a los ciudadanos por el Tratado de Lisboa. En este sentido, resul- 
ta especialmente importante la Carta de los Derechos Fundamentales jurídicamente vinculante y, en concreto, el artículo 41 relativo a una buena administración.

Ya en diciembre de 2009, como parte de nuestra contribución a la consulta pública sobre la reforma del Reglamento financiero de la Unión Europea $^{20}$, el Defensor del Pueblo subrayó que las normas pertinentes deberían tener en cuenta el artículo 41 y ofrecer orientaciones a los funcionarios sobre la forma de garantizar una buena gestión financiera y una buena administración. Por ejemplo, el Reglamento financiero debería, en circunstancias excepcionales, estipular pagos voluntarios como desagravio por las molestias graves o los daños psicológicos que pudiera ocasionar una mala administración. En mi opinión, la reforma del Reglamento financiero brinda una excelente oportunidad para aplicar el derecho fundamental a una buena administración.

Dado el elevado número de investigaciones sobre falta de transparencia que lleva a cabo el Defensor del Pueblo cada año, seguiré insistiendo en el derecho fundamental de acceso a los documentos, con arreglo a lo dispuesto en el artículo 42 de la Carta y el artículo 15 del TFUE.

Por último, procuraré garantizar que los ciudadanos, las asociaciones representativas y la sociedad civil se beneficien de las nuevas disposiciones del Tratado con respecto a la consulta, el diálogo y la posibilidad de expresar e intercambiar públicamente sus opiniones y contribuiré al éxito del nuevo y valioso instrumento para los ciudadanos que representa la iniciativa ciudadana.

\section{COLABORACIÓN CON LAS INSTITUCIONES PARA GARANTIZAR UNA CULTURA DE SERVICIO}

Parte de la función de un defensor del pueblo en cualquier democracia moderna es ocuparse de que la administración pública se oriente al servicio a los ciudadanos e intente satisfacer sus cada vez más elevadas expectativas. Esta idea queda reflejada en la declaración de misión del Defensor del Pueblo a la que he hecho referencia anteriormente. En mi calidad de Defensor del Pueblo, continuaré colaborando con las instituciones de la Unión para garantizar una cultura de servicio al ciudadano.

Sin embargo, con respecto dichas instituciones es importante señalar que no se constituyeron para tratar con los ciudadanos, como tampoco ofrecen los servicios públicos propios del Estado de bienestar. Esto significa que históricamente han carecido de estímulo para orientarse al ciudadano y a la cultura de servicio.

Por supuesto, las instituciones y su personal afirman, y, sin duda, consideran, que su actividad redunda en beneficio de los ciudadanos, pero esto no es lo mismo que comprometerse con ellos. Dicha situación puede y debería

20 El documento sobre la consulta de la Comisión puede consultarse en la siguiente dirección: http://ec.europa.eu/budget/library/consultations/FRconsult2009/consultation_paper_en.pdf 
cambiar. Como Defensor del Pueblo, me comprometo firmemente a facilitar este cambio. Un posible modelo en este sentido es el Banco Europeo de Inversión. Bajo la acertada dirección de su actual presidente, el Banco intenta conseguir un compromiso activo con las ONG, que suelen criticar en gran medida sus actividades.

Como ya he mencionado, el Tratado de Lisboa brinda a todas las instituciones la oportunidad de avanzar aún más en este sentido. Las disposiciones sobre el intercambio de opiniones y el mantenimiento de un diálogo pueden y deberían conllevar la búsqueda de un auténtico debate político con las organizaciones de la sociedad civil en el que puedan tanto criticar y rechazar las iniciativas de las instituciones como prestarles su apoyo.

Por otro lado, las instituciones deben esforzarse más por alentar a su propio personal a comportarse como ciudadanos activos en su lugar de trabajo y a mantener el contacto con sus Estados miembros mediante iniciativas como la denominada "Regreso a la escuela". Esto reportaría un doble beneficio: el personal de las instituciones conocería los puntos de vista y las experiencias de los ciudadanos y viceversa.

\section{PROMOCIÓN DE RECURSOS PARA LOS CIUDADANOS}

Un importante aspecto de mi labor en cuanto al refuerzo de la legitimidad de la Unión es promover recursos para los ciudadanos que tienen problemas para ejercer los derechos que les confiere el Derecho de la Unión. Los ciudadanos deberían disponer de recursos efectivos en caso de violación de sus derechos.

Dado que mi mandato se limita a la investigación de reclamaciones de mala administración relativas a instituciones europeas, no estoy facultado para conocer de reclamaciones contra los poderes públicos de los Estados miembros, ni siquiera cuando éstas se refieren a asuntos pertenecientes al ámbito de aplicación del Derecho de la Unión. Muchas de estas reclamaciones se inscriben dentro del ámbito de competencias de los defensores del pueblo nacionales y regionales de la Red Europea de Defensores del Pueblo. En este sentido, coopero estrechamente con mis compañeros de la Red para garantizar la correcta aplicación del Derecho de la Unión.

El Defensor del Pueblo nacional y los Defensores del Pueblo regionales de España son, por supuesto, miembros de esta Red y participan activamente en ella. La Red promueve tanto la buena administración del Derecho de la Unión a escala nacional como los recursos efectivos a escala local. Todos mis compañeros respaldan los principios en que se basa la Unión Europea ${ }^{21}$.

21 Véase la Declaración adoptada en el VI Seminario de Defensores del Pueblo nacionales que se celebró en Estrasburgo en octubre de 2007. Dicha Declaración se encuentra disponible en las veintitrés lenguas oficiales y puede consultarse en el portal del Defensor del Pueblo Europeo: http://www.ombudsman.europa.eu/liaison/es/statement.htm. 
Asimismo, se comprometen a tratar con imparcialidad, eficacia y equidad las reclamaciones relativas a actividades relacionadas con la Unión Europea que desempeñen las autoridades de los Estados miembros.

Espero poder reforzar la cooperación existente en la Red con el fin de asegurar que los ciudadanos puedan ejercer plenamente los derechos que les confiere el Derecho de la Unión. Por otro lado, procuraré garantizar la mejora de la calidad de la información facilitada a los ciudadanos sobre sus derechos y las vías de recurso disponibles. En concreto, promoveré la concienciación sobre el derecho de recurrir al Defensor del Pueblo y de formular peticiones al Parlamento (artículos 43 y 44 de la Carta, respectivamente), de forma que los ciudadanos puedan buscar mejor la reparación oportuna.

\section{CONCLUSIÓN}

La entrada en vigor del Tratado de Lisboa nos brinda una excelente oportunidad para fomentar la ciudadanía europea y capacitar a los ciudadanos frente a las instituciones de la Unión. El presente artículo ha intentado describir algunas de las muchas maneras en que puede alcanzarse este objetivo. No obstante, es importante mencionar que cualquier planteamiento centrado en la ciudadanía como medio para capacitar a los europeos debe tener en cuenta el hecho de que dicha capacitación debe producirse tanto ante las instituciones de la Unión como ante las autoridades nacionales en lo referente a asuntos de aquélla. No deberíamos descuidar la relación de los ciudadanos con las autoridades de su propio Estado miembro. Para la mayoría de las personas, su condición de ciudadanos europeos es importante sobre todo o exclusivamente en sus relaciones con las autoridades de su propio Estado miembro. Cualquier debate sobre la ciudadanía europea deberá tener en cuenta esta realidad.

Para capacitar a los ciudadanos europeos ante los encargados de ejercer el poder político es, en parte, necesario mejorar los mecanismos mediante los que pueden exigir responsabilidades a las autoridades nacionales por su aportación a las políticas y la legislación a escala europea y por su cumplimiento del Derecho de la Unión a escala nacional. Existen dos motivos principales que han llevado a desatender este importante aspecto hasta la fecha.

En primer lugar, el concepto de ciudadanía europea se ha entendido a menudo de modo que supone un juego de suma cero. Cuanto más se identifican las personas como ciudadanos europeos, menos importante se vuelve su ciudadanía nacional. Este planteamiento da poca cabida a la idea de que el proceso de integración europea podría exigir un enfoque centrado sistemáticamente en la responsabilidad de los poderes públicos nacionales ante los ciudadanos.

En segundo lugar, incluso cuando se ha entendido que la ciudadanía europea implica también relaciones con las autoridades de los Estados miembros, el planteamiento se ha centrado en los ciudadanos que tienen proble- 
mas con las autoridades de otro Estado miembro. Por ejemplo, podemos mencionar los tipos de problemas que surgen cuando los ciudadanos ejercen el derecho a la libertad de circulación.

Sin embargo, la Unión Europea y el Derecho europeo afectan cada vez más a las relaciones entre los ciudadanos y los poderes públicos de su país. Éste es el caso, por ejemplo, de la aplicación y el cumplimiento de las Directivas sobre discriminación, los derechos de los pasajeros aéreos y las tarifas de itinerancia de los teléfonos móviles.

A la vista de estos avances, queda claro que los poderes públicos de los Estados miembros deben tratar sistemáticamente a sus propios ciudadanos como ciudadanos europeos. A menos que esto ocurra, se prevé que un número cada vez mayor de personas percibirán la integración europea como un proceso que las excluye y desautoriza.

Especialmente en el actual período de crisis económica, debemos insistir en que la legitimidad de la Unión Europea no es un juego de suma cero, en el que las instituciones europeas pueden obtener beneficios a expensas de las instituciones políticas nacionales o viceversa. Por el contrario, sólo si colaboramos de forma que todos ganen —es decir, con eficacia, transparencia y ética- podrán las instituciones nacionales y de la Unión garantizar una responsabilidad y una participación plenas y, de este modo, reforzar conjuntamente su legitimidad ante los ciudadanos.

TitLE: The European Ombudsman and the Treaty of Lisbon.

ABSTRACT: The entry into force of the Treaty of Lisbon on December 1, 2009 marks the last phase of the process with recognition and training of the European citizens. One of the principal priorities to the European Ombudsman during the next five years will belong to help to guarantee that the European Union expires with the benefits promised to the citizens by the Treaty of Lisbon. The present article describes the repercussions that the Agreement will have in the labor of the European Ombudsman and centres on the most important innovations that the Agreement contributes for what it concerns the citizens.

Resumen: La entrada en vigor del Tratado de Lisboa el 1 de diciembre de 2009 marca la última fase del proceso en curso de reconocimiento y capacitación de los ciudadanos europeos. Una de las principales prioridades del Defensor del Pueblo Europeo durante los próximos cinco años será contribuir a garantizar que la Unión Europea cumpla con los beneficios prometidos a los ciudadanos por el Tratado de Lisboa. El presente artículo describe las repercusiones que tendrá el Tratado en la labor del Defensor del Pueblo Europeo y se centra en las novedades más importantes que aporta el Tratado por lo que respecta a los ciudadanos.

Palabras ClaVE: European Ombudsman. Treaty of Lisbon.

KeY wORDS: Defensor del Pueblo Europeo. Tratado de Lisboa.

FECHA DE RECEPCIÓN: 17.06.2010. FeCHA DE ACEPTACIÓN: 28.07.2010. 